\title{
Cosmic microwave background polarization in non-commutative space-time
}

\author{
S. Tizchang ${ }^{1}$, S. Batebi ${ }^{1}$, M. Haghighat ${ }^{2}$, R. Mohammadi $^{3, a}$ (i) \\ ${ }^{1}$ Department of Physics, Isfahan University of Technology, 84156-83111 Isfahan, Iran \\ ${ }^{2}$ Department of Physics, Shiraz University, Shiraz, Iran \\ ${ }^{3}$ Iran Science and Technology Museum (IRSTM), PO BOX: 11369-14611, Tehran, Iran
}

Received: 31 May 2016/ Accepted: 8 August 2016 / Published online: 30 August 2016

(C) The Author(s) 2016. This article is published with open access at Springerlink.com

\begin{abstract}
In the standard model of cosmology (SMC) the $\mathrm{B}$-mode polarization of the CMB can be explained by the gravitational effects in the inflation epoch. However, this is not the only way to explain the B-mode polarization for the $\mathrm{CMB}$. It can be shown that the Compton scattering in the presence of a background, besides generating a circularly polarized microwave, can lead to a B-mode polarization for the $\mathrm{CMB}$. Here we consider the non-commutative (NC) spacetime as a background to explore the CMB polarization at the last scattering surface. We obtain the B-mode spectrum of the $\mathrm{CMB}$ radiation by scalar perturbation of metric via a correction on the Compton scattering in NC-space-time in terms of the circular polarization power spectrum and the non-commutative energy scale. It can be shown that even for the $\mathrm{NC}$ scale as large as $20 \mathrm{TeV}$ the NC-effects on the CMB polarization and the $r$ parameter are significant. We show that the V-mode power spectrum can be obtained in terms of linearly polarized power spectrum in the range of microto nano-kelvin squared for the $\mathrm{NC}$ scale of about $1-20 \mathrm{TeV}$, respectively.
\end{abstract}

\section{Introduction}

The polarization anisotropy and temperature inhomogeneities of the cosmic microwave background radiation (CMBR) can provide a way to explore the physics of the early universe. The light polarization can be parameterized in terms of the Stokes parameters $(Q, U$, and $V)$. Nonzero values for $Q$ and/or $U$ show linearly polarized radiation, while a circular polarized radiation has a non-vanishing value for the Stokes parameter $V$ [1]. An anisotropic Thomson scattering due to the temperature inhomogeneity around the recombination phase leads to the linear polarization of about $10 \%$ of

a e-mail: rmohammadi@ipm.ir
CMBR [2,3]. Meanwhile, according to the standard model of cosmology there is no physical mechanism to generate circular polarized radiation at the last scattering surface or $V=0$. However, linearly polarized radiation through its propagation in a magnetic field can be partially circular polarized, a property known as the Faraday effect. The Stokes parameter $V$ in this mechanism evolves as

$\dot{V}=2 U \frac{\mathrm{d} \Delta \phi_{\mathrm{FC}}}{\mathrm{d} t}$,

where $\Delta \phi_{\mathrm{FC}}$ is the Faraday conversion phase shift [4]. Linear polarization of the $\mathrm{CMB}$ radiation from the last scattering can be converted to circular polarization due to the effects of background fields, particle scattering, and temperature fluctuations. The conversion probability of the CMB linear polarization to the circular polarization has been discussed in many papers [5-11]. Furthermore, since $Q$ and $U$ are framedependent, by decomposing the linear polarization into the $E$ and $B$ components one can extract more information from the polarization pattern on the sky. Thomson scattering at the last scattering surface only produces the $E$ mode, which can be converted to the $B$ mode through vector and tensor perturbations. Meanwhile, the gravitational waves, due to the tensor mode perturbation arising from the inflation epoch, generate the $\mathrm{B}$-mode polarization for the $\mathrm{CMB}$ radiation.

In early 2014, the BICEP2 team announced a nonzero measurement on the $\mathrm{B}$-mode polarization for the $\mathrm{CMB}$ radiation as evidence for the primordial gravitational wave [12]. This result is not consistent with the Planck limit, $r<$ $0.11(98 \% \mathrm{CL})$. However, at this time there is no conclusive evidence of primordial gravitational waves from a joint analysis of data provided by the Planck and BICEP 2 experiments. The recent Bicep/Keck Array observation reported upper bounds on the tensor-to-scalar ratio, $r_{0.05}<0.09$ and $r_{0.05}<0.07$ at $(95 \%)$ CL by using B modes alone and combining the B-mode results with the Planck temperature 
analysis, respectively [13-18]. In fact, to distinguish the tensor and scalar components, the tensor-to-scalar ratio can be calculated by measuring the polarization angles on the sky; Plank has reported this ratio to be about $r \sim 0.12$. Therefore, to find the contribution of the gravitational wave on the $B$ mode one should consider all contributions from the other sources. Although in the standard model of cosmology the $\mathrm{B}$ mode does not receive a contribution from the scalar mode, one can consider the B mode as a result of Faraday rotation of the E-mode polarization [6,13-19]. Furthermore, the Compton scattering in the presence of a background can potentially lead to a B-mode polarization for the CMB even for the scalar perturbation. The contribution to the observed B-mode spectrum from the interaction between CMB photon and the cosmic neutrino background (CNB) in the scalar perturbation background has been considered in [20,21].

Here we would like to explore the effects of noncommutative background on the B-mode polarization. In Ref. [22] the energy scale of the non-commutativity of spacetime has been constrained by using CMB data from Planck. They found that Planck data put the lower bound on the non-commutativity energy scale to about $20 \mathrm{TeV}$, which is about a factor of 2 larger than the previous bound, which was obtained using data from WMAP, ACBAR, and CBI.

In this paper we study the possibility of generating circular polarization of $\mathrm{CMB}$ radiation by considering Compton scattering on non-commutative background. In Sect. 2 we review the Stokes parameters and the formalism of the Boltzmann equation. In Sect. 3 we give a brief introduction of the noncommutative standard model. In Sect. 4 the time evolution of the Stokes parameters by using the scalar mode perturbation of metric and the generation of circular polarization on non-commutative space is computed. Then we calculate the circular, E- and B-mode spectra of the CMB. By comparing our results with experimental data the lower limit of the non-commutative energy scale is obtained.

\section{Stokes parameters and Boltzmann equation}

For a monochromatic electromagnetic wave propagating in the $\hat{z}$ direction, the electric field components can be given as

$E_{x}=a_{x} \cos \left(\omega t-\theta_{x}\right), \quad E_{y}=a_{x} \cos \left(\omega t-\theta_{y}\right)$,

where $a_{x}$ and $a_{y}$ are the amplitudes and $\theta_{x}$ and $\theta_{y}$ are the phase angles. The electromagnetic field can be parameterized in terms of the Stokes parameters

$I=\left\langle a_{x}^{2}\right\rangle+\left\langle a_{y}^{2}\right\rangle$

which is the total intensity and

$Q=\left\langle a_{x}^{2}\right\rangle-\left\langle a_{y}^{2}\right\rangle, \quad ; \quad U=\left\langle 2 a_{x} a_{y} \cos \left(\theta_{x}-\theta_{y}\right)\right\rangle$, where the $Q$ and $U$ parameters indicate the intensities of the linear polarizations of the electromagnetic waves, and the Stokes parameter for the circular polarization is

$V=\left\langle 2 a_{x} a_{y} \sin \left(\theta_{x}-\theta_{y}\right)\right\rangle$.

One can see that under a right-handed rotation of the coordinate axes perpendicular to the direction $\hat{n}$ on the sky, $Q$ and $U$ with a rotation's angle $\psi$ transform to

$Q^{\prime}=Q \cos (2 \psi)+U \sin (2 \psi)$,

$U^{\prime}=-Q \sin (2 \psi)+U \cos (2 \psi)$,

and the Stokes parameters $I$ and $V$ remain unchanged. The density matrix in terms of the Stokes parameters is defined as

$\rho=\frac{1}{2}\left(\begin{array}{ll}I+Q & U-i V \\ U+i V & I-Q\end{array}\right)$.

Meanwhile, a system of photons can be described by the density operator [2]

$\hat{\rho}=\frac{1}{\mathrm{t} r}(\hat{\rho}) \int \frac{\mathrm{d}^{3} \mathbf{k}}{(2 \pi)^{3}} \rho_{i j}(\mathbf{k}) a_{i}^{\dagger}(\mathbf{k}) a_{j}(\mathbf{k})$,

where $\rho_{i j}(\mathbf{k})$ is the general density matrix, which is related to the photon number operator $D_{i j}^{0}(\mathbf{k}) \equiv a_{i}^{\dagger}(\mathbf{k}) a_{j}(\mathbf{k})$. The canonical commutation relations of the creation and annihilation operators for photons and electrons are defined as

$\left[a_{s}(p), a_{s^{\prime}}^{\dagger}\left(p^{\prime}\right)\right]=(2 \pi)^{3} 2 p^{0} \delta^{3}\left(\mathbf{p}-\mathbf{p}^{\prime}\right) \delta_{s s^{\prime}}$,

$\left\{b_{r}(q), b_{r^{\prime}}^{\dagger}\left(q^{\prime}\right)\right\}=(2 \pi)^{3} \frac{q^{0}}{m} \delta^{3}\left(\mathbf{q}-\mathbf{q}^{\prime}\right) \delta_{r r^{\prime}}$,

where $s$ and $r$ show the photon polarization and electron spin while the bold and plain momenta represent the three dimensional vectors and the four-momentum vectors, respectively. For the expectation value of the number operator one has

$\left\langle D_{i j}^{0}(\mathbf{k})\right\rangle \equiv \operatorname{tr}\left[\hat{\rho} D_{i j}^{0}(\mathbf{k})\right]=(2 \pi)^{3} \delta^{3}(0)\left(2 k^{0}\right) \rho_{i j}(\mathbf{k})$,

and in the Heisenberg picture, the time evolution of the operator $D_{i j}^{0}(\mathbf{k})$ can be obtained:

$\frac{\mathrm{d}}{\mathrm{d} t} D_{i j}^{0}(\mathbf{k})=i\left[H, D_{i j}^{0}(\mathbf{k})\right]$

where $H$ is the total Hamiltonian. Therefore, the time evolution of the density matrix can be written as follows:

$$
\begin{aligned}
& (2 \pi)^{3} \delta^{3}(0)\left(2 k^{0}\right) \frac{\mathrm{d}}{\mathrm{d} t} \rho_{i j}(\mathbf{k}) \\
& \quad=i\left\langle\left[H_{I}^{0}(t) ; D_{i j}^{0}(\mathbf{k})\right]\right\rangle-\frac{1}{2} \int \mathrm{d} t\left\langle\left[H_{I}^{0}(t) ;\left[H_{I}^{0}(0) ; D_{i j}^{0}(\mathbf{k})\right]\right]\right\rangle,
\end{aligned}
$$

where $H_{I}^{0}(t)$ is the interacting Hamiltonian at the lowest order. The first term on the right-hand side of (12) is a forward 
scattering term, while the second term is the higher-order collision term so-called damping term. To calculate the righthand side of (12) one needs the operator expectation values,

$\left\langle a_{1} a_{2} \cdots b_{1} b_{2} \cdots\right\rangle=\left\langle a_{1} a_{2} \cdots\right\rangle\left\langle b_{1} b_{2} \cdots\right\rangle$,

where for the two point functions using the commutation relations (9) one has

$\left\langle a_{m}^{\dagger}\left(p^{\prime}\right) a_{n}(p)\right\rangle=2 p^{0}(2 \pi)^{3} \delta^{3}\left(\mathbf{p}-\mathbf{p}^{\prime}\right) \rho_{m n}(\mathbf{p})$

and

$\left\langle b_{m}^{\dagger}\left(q^{\prime}\right) b_{n}(q)\right\rangle=\frac{q^{0}}{m}(2 \pi)^{3} \delta^{3}\left(\mathbf{q}-\mathbf{q}^{\prime}\right) \delta_{m n} \frac{1}{2} n_{f}(\mathbf{q})$,

where $n_{f}(\mathbf{q})$ represents the number density of fermions (electrons and protons) with momentum q per unit volume. Meanwhile, the distributions of the fermions in $x$ space are defined by

$n_{f}(\mathbf{x})=\int \frac{\mathrm{d}^{3} \mathbf{q}}{(2 \pi)^{3}} n_{f}(\mathbf{x}, \mathbf{q})$,

$m_{f} v_{i}(\mathbf{x}) n_{f}(\mathbf{x})=\int \frac{\mathrm{d}^{3} \mathbf{q}}{(2 \pi)^{3}} q_{i} n_{f}(\mathbf{x}, \mathbf{q})$.

\section{Non-commutative standard model}

The non-commutative space-time is one of the consequences of the string theory. Indeed, as predicted in [23] and shown by Seiberg and Witten [24], the endpoints of an open string on a D-brane in a constant B-field background live on a noncommutative space-time. In the canonical form one has

$\theta^{\mu \nu}=-i\left[\hat{x}^{\mu}, \hat{x}^{\nu}\right]$,

where $\theta^{\mu \nu}$ is a real, constant, and antisymmetric matrix; $\theta^{\mu \nu} \propto 1 / \Lambda_{\mathrm{NC}}^{2}$, and $\Lambda_{\mathrm{NC}}$ is the non-commutative scale. The non-commutative parameter, $\theta^{\mu \nu}$, can be divided into

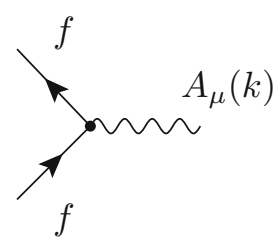

$$
\begin{aligned}
i e Q_{f} & {\left[\gamma_{\mu}-\frac{i}{2} k^{\nu}\left(\theta_{\mu \nu \rho} p_{\mathrm{in}}^{\rho}-\theta_{\mu \nu} m_{f}\right)\right] } \\
= & i e Q_{f} \gamma_{\mu} \\
& +\frac{1}{2} e Q_{f}\left[\left(p_{\text {out }} \theta p_{\text {in }}\right) \gamma_{\mu}-\left(p_{\text {out }} \theta\right)_{\mu}\left(\gamma \lambda p_{\text {in }}^{\lambda}-m_{f}\right)-\left(\gamma \lambda p_{\text {out }}^{\lambda}-m_{f}\right)\left(\theta p_{\text {in }}\right)_{\mu}\right] .
\end{aligned}
$$

two parts: the time-space components $\left(\theta^{01}, \theta^{02}, \theta^{03}\right)$, which denote the electric-like part and the magnetic-like part, which contains the space-space components $\left(\theta^{23}, \theta^{31}, \theta^{12}\right)$. To the ordinary products between fields in the corresponding commutative versions with the Moyal- $\star$ product, which is defined as follows:

$(f \star g)(x)=f(x) \exp \left(\frac{i}{2} \overleftarrow{\partial_{\mu}} \theta^{\mu \nu} \overrightarrow{\partial_{\nu}}\right) g(x)$

By applying this correspondence, there would be two approaches to construct the non-commutative standard model. The first one is based on the Moyal- $\star$ product and the Seiberg-Witten maps in which the gauge group is $\mathrm{SU}(3) \times \mathrm{SU}(2)_{L} \times U(1)_{Y}$, the numbers of particles and gauge fields are the same as the ordinary standard model [25]. Furthermore, matter fields, gauge fields, and gauge parameters should be expanded via the Seiberg-Witten map as a power series of $\theta$ [25] in terms of the commutative fields,

$$
\begin{aligned}
\widehat{\psi} & =\psi+\frac{1}{2} \theta_{\mu \nu} A_{\nu} \partial_{\mu} \psi+\mathcal{O}\left(\theta^{2}\right), \\
\widehat{A}_{\mu} & =A_{\mu}+\frac{1}{4} \theta^{\rho v}\left\{A_{\nu},\left(\partial_{\rho} A_{\mu}+F_{\rho \mu}\right)\right\}+\mathcal{O}\left(\theta^{2}\right), \\
\widehat{\Lambda} & =\Lambda+\frac{1}{4} \theta^{\mu \nu}\left\{A_{\nu}, \partial_{\mu} \Lambda\right\}+\mathcal{O}\left(\theta^{2}\right),
\end{aligned}
$$

where the hats show the non-commutative fields which reduce to their counterparts in the ordinary space in the limit $\theta \rightarrow 0$.

In the second approach the gauge group is $U(3) \times U(2) \times$ $U(1)$, which is reduced to $\mathrm{SU}(3) \times \mathrm{SU}(2)_{L} \times U(1)_{Y}$ by an appropriate symmetry breaking [26-29]. In both versions, besides corrections on the usual standard model interactions, many new interactions would appear. For instance, in the QED part of the NCSM through the first approach there is a correction on the photon-fermion vertex $f f \gamma$, which can be derived up to the first order of $\theta$ as [30] construct non-commutative field theories, one should replace 
Meanwhile, in the non-commutative QED there are vertices which have not any counterpart in the ordinary QED. For example, two photons can directly couple to two fermions in $\mathrm{NC}$ space as follows:

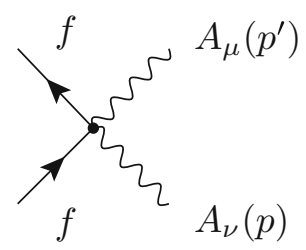

$$
\frac{-e^{2} Q_{f}^{2}}{2} \theta_{\mu \nu \rho}\left(p^{\rho}-p^{\rho}\right),
$$

where photons momenta are taken to be incoming and $\theta^{\mu \nu \rho}$ is a totally antisymmetric quantity which is defined as

$\theta^{\mu \nu \rho}=\theta^{\mu \nu} \gamma^{\rho}+\theta^{\nu \rho} \gamma^{\mu}+\theta^{\rho \mu} \gamma^{\nu}$.

\section{Compton scattering in NC space-time}

The photons in the cosmic microwave background can be scattered from all charged particles with the scattering rate proportional to the inverse mass squared. Therefore, in the ordinary space as a good approximation the Compton scattering on the electrons is usually considered. There are five diagrams in the NCSM to describe the Compton scattering on non-commutative space-time. By replacing the ordinary couplings with the NC vertices, four diagrams can be obtained, which is shown in Fig. 1. The fifth diagram in which two fermions directly couple to two photons is given in (21). Therefore, the amplitude of the Compton scattering in the NCQED can be given as

$\mathcal{M}_{\mathrm{NCQED}}=\mathcal{M}_{\mathrm{QED}}+\mathcal{M}^{\theta}$,

where

$$
\begin{aligned}
i \mathcal{M}^{\theta}= & i \mathcal{M}_{1}^{\theta}+i \mathcal{M}_{2}^{\theta}+i \mathcal{M}_{3}^{\theta}+i \mathcal{M}_{4}^{\theta}+i \mathcal{M}_{5}^{\theta} \\
= & \frac{e^{2} Q_{f}^{2}}{4 p \cdot q} \bar{u}_{\dot{r}}\left(q^{\prime}\right)\left[\left(k_{s}(\dot{p}) \dot{q} \cdot \theta \cdot(q+p)\right.\right. \\
& \left.\left.-\dot{q} \cdot \theta \cdot \varepsilon_{\dot{s}}(\dot{p}) \not p\right)(q)+\not p+m_{f}\right) \phi_{s}(p) \\
& +\xi_{\dot{s}}(\dot{p})\left(\not q+\not p+m_{f}\right)\left(\phi_{s}(p)(q+p) \cdot \theta \cdot q\right. \\
& \left.\left.-q \cdot \theta \cdot \varepsilon_{s}(p) \not p\right)\right] u_{r}(q) \\
& -\frac{e^{2} Q_{f}^{2}}{4 \dot{p} \cdot q} \bar{u}_{\dot{r}}(\dot{q})\left[\left(\phi_{s}(p) \dot{q} \cdot \theta \cdot(q-\dot{p})\right.\right.
\end{aligned}
$$

$$
\begin{aligned}
& \left.+\dot{q} \cdot \theta \cdot \varepsilon_{s}(p) \not p\right)\left(q-\not p^{\prime}+m\right) \xi_{\dot{s}}(\dot{p}) \\
& +\phi_{S}(p)\left(q-\not p+m_{f}\right) \\
& \left.\times\left(\varepsilon_{\dot{s}}(\dot{p})\left(q-p^{\prime}\right) \cdot \theta \cdot q+q \cdot \theta \cdot \varepsilon_{\dot{s}}(\dot{p}) \not p\right)\right] u_{r}(q) \\
& -\frac{e^{2} Q_{f}^{2}}{2} \bar{u}_{\dot{r}}\left(q^{\prime}\right) \varepsilon_{\dot{s}}^{\mu}\left(p^{\prime}\right)\left(p+p^{\prime}\right)^{\rho} \theta_{\mu \nu \rho} \varepsilon_{s}^{\nu}(p) u_{r}(q)
\end{aligned}
$$

and $p \cdot \theta \cdot q \equiv p_{\mu} \theta^{\mu \nu} q_{\nu}$. Now the leading-order interacting Hamiltonian can be obtained as follows:

$$
\begin{aligned}
H_{I}^{0}= & \int \operatorname{dq} \mathbf{q} \mathrm{d} \mathbf{q}^{\prime} \mathrm{d} \mathbf{p} \mathrm{d} \mathbf{p}^{\prime}(2 \pi)^{3} \delta^{3}\left(\mathbf{q}^{\prime}+\mathbf{p}^{\prime}-\mathbf{p}-\mathbf{q}\right) \\
& \times \exp \left[\operatorname{it}\left(q^{\prime 0}+p^{\prime 0}-q^{0}-p^{0}\right)\right]\left[b_{r^{\prime}}^{\dagger} a_{s^{\prime}}^{\dagger}\left(\mathcal{M}^{\theta}\right) a_{s} b_{r}\right],
\end{aligned}
$$

where $\mathrm{d} \mathbf{q} \equiv \frac{\mathrm{d}^{3} q}{(2 \pi)^{3}} \frac{m_{f}}{q^{0}}$ and $\mathrm{d} \mathbf{p} \equiv \frac{\mathrm{d}^{3} p}{(2 \pi)^{3} 2 p^{0}}$, and we have similar expressions for $\mathrm{d} \mathbf{q}^{\prime}$ and $\mathrm{d} \mathbf{p}^{\prime}$, respectively.

\section{Density matrix elements and CMB Polarization on NC space-time}

In the preceding section we found the amplitude for Compton scattering in the NCQED. Now substituting the NC interacting Hamiltonian (25) in (12) leads to

$$
\begin{aligned}
i & \left\langle\left[\hat{H}_{I}(0), \mathcal{D}_{i j}(\mathbf{k})\right]\right\rangle=-\frac{e^{2} Q_{f}^{2}}{2} \delta^{3}(0) \\
& \times \int \mathrm{d} \mathbf{q}(2 \pi)^{3} n_{f}(\mathbf{x}, \mathbf{q})\left(\delta_{i s} \rho_{s^{\prime} j}(\mathbf{k})\right. \\
& \left.-\delta_{j s^{\prime}} \rho_{i s}(\mathbf{k})\right) \bar{u}_{r}(q) \\
& \times\left\{\frac { 1 } { 4 k \cdot q } \left[q \cdot \theta \cdot \varepsilon _ { s ^ { \prime } } ( k ) \left(k\left(\not q+\not k+m_{f}\right) \phi_{s}(k)\right.\right.\right. \\
& \left.+\phi_{s}(k)\left(\not q-\not k+m_{f}\right) \not k\right) \\
& +q \cdot \theta \cdot \varepsilon_{S}(k)\left(\varepsilon_{s^{\prime}}(k)\left(q+\not k+m_{f}\right) k\right. \\
& \left.\left.+k\left(q-\not k+m_{f}\right) \phi_{s^{\prime}}(k)\right)\right] \\
& +\left[\varepsilon_{s^{\prime}}(k) \cdot \theta \cdot \varepsilon_{s}(k) k-k \cdot \theta \cdot \epsilon_{s}(k) \xi_{s}(k)\right. \\
& \left.\left.+k \cdot \theta \cdot \varepsilon_{s^{\prime}}(k) \phi_{s}(k)\right]\right\} u_{r}(q) .
\end{aligned}
$$
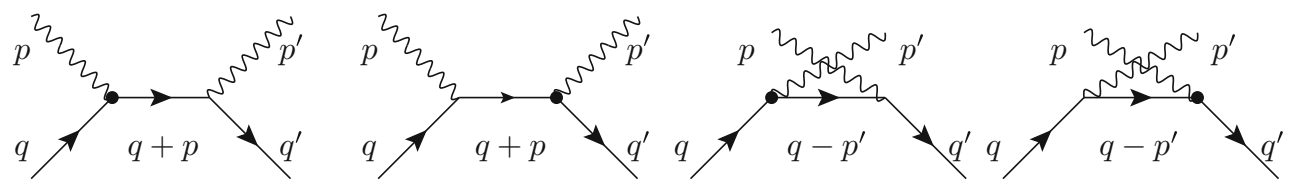

Fig. 1 Compton scattering in NC space 
Therefore, the time evaluation of the density matrix after a little algebra can be obtained:

$$
\begin{aligned}
\frac{\mathrm{d}}{\mathrm{d} t} \rho_{i j}(\mathbf{k})= & -\frac{e^{2} Q_{f}^{2}}{4 m_{f} k^{0}} \int \mathrm{d} \mathbf{q} n_{f}(\mathbf{x}, \mathbf{q})\left(\delta_{i s} \rho_{s j}-\delta_{j s} \rho_{i s}\right) \\
& \times(A+B),
\end{aligned}
$$

where

$A=q \cdot \theta \cdot \varepsilon_{s}^{\prime} q \cdot \varepsilon_{s}+q \cdot \theta \cdot \varepsilon_{s} q \cdot \varepsilon_{s}^{\prime}$

and

$B=\left(\varepsilon_{s^{\prime}} \cdot \theta \cdot \varepsilon_{s} k \cdot q-k \cdot \theta \cdot \varepsilon_{S} \quad \varepsilon_{s^{\prime}} \cdot q+k \cdot \theta \cdot \varepsilon_{S^{\prime}} \varepsilon_{s} \cdot q\right)$.

It should be noted that $A$ and $B$ are related to the NC contribution on the time evolution of the density matrix from the vertices given in (20) and (21), respectively. As we show below the first contribution depends on the time-like component of the NC parameter $\theta^{0 i}$, while the second contribution depends on the space-like component of the NC parameter $\theta^{i j}$. To this end we individually investigate the contribution of each part on the time evolution of the Stokes parameters as follows.

Part A: For the $A$ term of (27) which is due to the diagrams given in Fig. 1 the time evolution of the density matrix is

$$
\frac{\mathrm{d}}{\mathrm{d} t} \rho_{i j}(\mathbf{k})=-\frac{e^{2} Q_{f}^{2}}{4 m_{f} k^{0}} \int \mathrm{d} \mathbf{q} n_{f}(\mathbf{x}, \mathbf{q})\left(\delta_{i s} \rho_{s j}-\delta_{j \bar{s}} \rho_{i s}\right) A .
$$

By using Eq. (16) and assuming

$\theta^{01}=\theta^{02}=\theta^{03}=\frac{1}{\Lambda^{2}}, \quad \hat{\theta}^{0 i}=\Lambda^{2} \theta^{0 i}$,

and

$\theta^{i j}=\frac{\hat{\theta}^{i j}}{\Lambda^{2}}, \quad \hat{\theta}^{i j}=\Lambda^{2} \theta^{i j}$,

where $\theta^{i j} ; i, j \in\{1,2,3\}$ are the space-like components of the $\mathrm{NC}$ parameter. One can rewrite (30) as follows:

$$
\begin{aligned}
\frac{\mathrm{d}}{\mathrm{d} t} \rho_{i j}(\mathbf{k})= & \frac{e^{2} Q_{f}^{2}}{4} \frac{m_{f}}{k^{0}} \frac{1}{\Lambda^{2}} \bar{n}_{f}(\mathbf{x})\left(\delta_{i s} \rho_{s j}-\delta_{j \bar{s}} \rho_{i s}\right) \\
& \times\left[\left(\hat{\theta}^{0 i} \varepsilon_{s i} v_{f} \cdot \varepsilon_{s}+\hat{\theta}^{0 i} \varepsilon_{s i} v_{f} \cdot \varepsilon_{\dot{s}}\right)+\bigcirc\left(v_{f}^{2}\right)\right],
\end{aligned}
$$

where the polarization four-vectors $\varepsilon_{\mu i}(k)$, with $i, j, s$ and $s^{\prime}$ running over 1,2 , represent two transverse polarizations of the photon, $\bar{n}_{f}$ represents the number density of fermions, and $v_{f}$ is the fermion bulk velocity which is a small quantity. Note that the second term in the second line of (33) depends on $v_{f}^{2}$, which is negligible in comparison with the first term.
Therefore, the time derivative of the components of the density matrix can be cast into

$$
\begin{aligned}
\frac{\mathrm{d}}{\mathrm{d} t} \rho_{11}(\mathbf{k})= & \frac{3}{8} \frac{m_{f}}{k^{0}} \frac{\sigma^{T}}{\alpha} \frac{m_{e}^{2}}{\Lambda^{2}} \bar{n}_{f} \hat{\theta}^{0 i} \\
& \times\left(\varepsilon_{2 i} v_{f} \cdot \varepsilon_{1}+\varepsilon_{1 i} v_{f} \cdot \varepsilon_{2}\right)\left(\rho_{21}-\rho_{12}\right),
\end{aligned}
$$

$$
\begin{aligned}
\frac{\mathrm{d}}{\mathrm{d} t} \rho_{22}(\mathbf{k})= & \frac{3}{8} \frac{m_{f}}{k^{0}} \frac{\sigma^{T}}{\alpha} \frac{m_{e}^{2}}{\Lambda^{2}} \bar{n}_{f} \hat{\theta}^{0 i} \\
& \times\left(\varepsilon_{2 i} v_{f} \cdot \varepsilon_{1}+\varepsilon_{1 i} v_{f} \cdot \varepsilon_{2}\right)\left(\rho_{12}-\rho_{21}\right),
\end{aligned}
$$

$$
\begin{aligned}
\frac{\mathrm{d}}{\mathrm{d} t} \rho_{12}(\mathbf{k})= & \frac{3}{8} \frac{m_{f}}{k^{0}} \frac{\sigma^{T}}{\alpha} \frac{m_{e}^{2}}{\Lambda^{2}} \bar{n}_{f} \hat{\theta}^{0 i} \\
& \times\left(2\left(\varepsilon_{1 i} v_{f} \cdot \varepsilon_{1}-\varepsilon_{2 i} v_{f} \cdot \varepsilon_{2}\right) \rho_{12}\right. \\
& -\left(\varepsilon_{2 i} v_{f} \cdot \varepsilon_{1}+\varepsilon_{1 i} v_{f} \cdot \varepsilon_{2}\right) \rho_{11} \\
& \left.+\left(\varepsilon_{2 i} v_{f} \cdot \varepsilon_{1}+\varepsilon_{1 i} v_{f} \cdot \varepsilon_{2}\right) \rho_{22}\right),
\end{aligned}
$$

$$
\begin{aligned}
\frac{\mathrm{d}}{\mathrm{d} t} \rho_{21}(\mathbf{k})= & -\frac{3}{8} \frac{m_{f}}{k^{0}} \frac{\sigma^{T}}{\alpha} \frac{m_{e}^{2}}{\Lambda^{2}} \bar{n}_{f} \hat{\theta}^{0 i} \\
& \times\left(2\left(\varepsilon_{1 i} v_{f} \cdot \varepsilon_{1}-\varepsilon_{2 i} v_{f} \cdot \varepsilon_{2}\right) \rho_{21}\right. \\
& -\left(\varepsilon_{2 i} v_{f} \cdot \varepsilon_{1}+\varepsilon_{1 i} v_{f} \cdot \varepsilon_{2}\right) \rho_{11} \\
& \left.+\left(\varepsilon_{2 i} v_{f} \cdot \varepsilon_{1}+\varepsilon_{1 i} v_{f} \cdot \varepsilon_{2}\right) \rho_{22}\right),
\end{aligned}
$$

where $m_{e}$ is the mass of electron, $\sigma^{T}$ is the Thomson cross section, $\alpha=e^{2} / 4 \pi$ and $Q_{f}^{2}=1$. Using the density operator matrix elements, the time variation of the Stokes parameters, the linear polarization intensities $Q$ and $U$ and the difference between the left- and right-handed polarization $V$ in the NC space can be obtained as follows:

$\dot{V}(\mathbf{k})=i \frac{3}{4} \frac{m_{f}}{k^{0}} \frac{\sigma^{T}}{\alpha} \frac{m_{e}^{2}}{\Lambda^{2}} \bar{n}_{f}(C Q+D U)$,

$\dot{Q}(\mathbf{k})=i \frac{3}{4} \frac{m_{f}}{k^{0}} \frac{\sigma^{T}}{\alpha} \frac{m_{e}^{2}}{\Lambda^{2}} \bar{n}_{f}(-C V)$,

$\dot{U}(\mathbf{k})=i \frac{3}{4} \frac{m_{f}}{k^{0}} \frac{\sigma^{T}}{\alpha} \frac{m_{e}^{2}}{\Lambda^{2}} \bar{n}_{f}(-D V) ;$

where

$$
\begin{aligned}
& C=-\hat{\theta}^{0 i}\left(\varepsilon_{1 i} v_{f} \cdot \varepsilon_{2}+\varepsilon_{2 i} v_{f} \cdot \varepsilon_{1}\right), \\
& D=\hat{\theta}^{0 i}\left(\varepsilon_{1 i} v_{f} \cdot \varepsilon_{1}-\varepsilon_{2 i} v_{f} \cdot \varepsilon_{2}\right) .
\end{aligned}
$$

These equations show that the contribution of the $A$ term to the time evolution of the Stokes parameters depends on the mass and bulk velocity of fermion and the time-like components of the NC parameter $\theta^{0 i}$, and as already claimed there is not any contribution from the space-space part of the $\mathrm{NC}$ parameter. In contrast with the usual Compton scattering, which has a larger cross section for particles with lower masses, the evolution of the Stokes parameters in the NC space is directly proportional to the fermion masses, which 
leads to the larger values for the scattering from fermions with larger masses; see Eqs. (38)-(40). In fact, since the average number of electrons $\bar{n}_{e}$ approximately equals the average number of protons $\bar{n}_{p}$ due to electric neutrality in cosmology, in the NC space-time the contribution to the evolution of the Stokes parameters of photon-proton forward scattering is larger than photon-electron scattering by a factor $m_{p} / m_{e}$. Nevertheless, to have any significant effects from the $A$ term on the CMB polarization, the factor $\frac{3}{8} \frac{m_{f}}{k^{0}} \frac{1}{\alpha} \frac{m_{e}^{2}}{\Lambda^{2}}$ should be comparable to one. It should be noted that $\frac{m_{f}}{k^{0}}$ is much larger than unity, which may compensate the smallness of $\frac{m_{e}^{2}}{\Lambda^{2}}$.

Part B: For the $B$ term of (27), which comes from the direct vertex (21), we have

$$
\begin{aligned}
\frac{\mathrm{d}}{\mathrm{d} t} \rho_{11}(\mathbf{k})= & -\frac{\mathrm{d}}{\mathrm{d} t} \rho_{22}(\mathbf{k})=-\frac{e^{2}}{4 m_{f} k^{0}} \\
& \times \int \mathrm{d} \mathbf{q} n_{f}(\mathbf{x}, \mathbf{q})\left(\rho_{12}+\rho_{21}\right) \\
& \times\left[\varepsilon_{2} \cdot \theta \cdot \varepsilon_{1} k \cdot q-k \cdot \theta \cdot \varepsilon_{1} \varepsilon_{2} \cdot q\right. \\
& \left.+k \cdot \theta \cdot \varepsilon_{2} \varepsilon_{1} \cdot q\right], \\
\frac{\mathrm{d}}{\mathrm{d} t} \rho_{12}(\mathbf{k})= & \frac{\mathrm{d}}{\mathrm{d} t} \rho_{21}(\mathbf{k})=-\frac{e^{2}}{4 m_{f} k^{0}} \\
& \times \int \mathrm{d} \mathbf{q} n_{f}(\mathbf{x}, \mathbf{q})\left(\rho_{22}-\rho_{11}\right) \\
& \times\left[\varepsilon_{2} \cdot \theta \cdot \varepsilon_{1} k \cdot q-k \cdot \theta \cdot \varepsilon_{1} \varepsilon_{2} \cdot q\right. \\
& \left.+k \cdot \theta \cdot \varepsilon_{2} \varepsilon_{1} \cdot q\right],
\end{aligned}
$$

which, after some calculations, leads to

$$
\begin{aligned}
\dot{I}(\mathbf{k}) & =0, \quad \dot{V}(\mathbf{k})=0 \\
\dot{Q}(\mathbf{k}) \pm i \dot{U}(\mathbf{k}) & = \pm 2 i F(Q(\mathbf{k}) \pm i U(\mathbf{k}))
\end{aligned}
$$

where

$$
\begin{aligned}
F= & \left.\sigma\right|_{\mathrm{NCd} v} \bar{n}_{f}\left\{\hat{\theta}^{0 i}\left(\varepsilon_{1 i} v_{f} \cdot \varepsilon_{2}-\varepsilon_{2 i} v_{f} \cdot \varepsilon_{1}\right)\right. \\
& +\left[\varepsilon_{2} \cdot \hat{\theta} \cdot \varepsilon_{1}\left(1-v_{f} \cdot \hat{k}\right)\right. \\
& \left.\left.+\hat{k} \cdot \hat{\theta} \cdot \varepsilon_{1} \quad v_{f} \cdot \varepsilon_{2}-\hat{k} \cdot \hat{\theta} \cdot \varepsilon_{2} v_{f} \cdot \varepsilon_{1}\right]\right\}
\end{aligned}
$$

and

$\left.\sigma\right|_{\mathrm{NCd} v}=\frac{3}{8} \frac{\sigma_{T}}{\alpha} \frac{m_{e}^{2}}{\Lambda^{2}}$.

Here the time evolution of the Stokes parameters depends on the space-space part of the NC parameter as well. However, in order to have any significant effect on the CMB polarization, the value of $\left.\sigma\right|_{\mathrm{NCd} v}$ should be comparable to $\sigma_{T}$. For photon-proton scattering one has
$\left.\sigma\right|_{\mathrm{NCd} v} / \sigma_{T} \propto \frac{1}{\alpha}\left(\frac{m_{e}}{\Lambda}\right)^{2}<10^{-10}\left(\frac{\mathrm{TeV}}{\Lambda}\right)^{2}$

which is too small to be considered. Therefore, we can neglect the $B$ term with respect to the $A$ term in evaluating the CMB polarization in the next sections.

\section{Time evolution of polarized CMB photons}

In this section we expand the primordial scalar perturbations $(S)$ in the Fourier modes, which are characterized by a wavenumber $\mathbf{K}$. For a given Fourier mode $\mathbf{K}$, one can select a coordinate system where $\mathbf{K} \| \hat{\mathbf{z}}$ and $\left(\hat{\mathbf{e}}_{1}, \hat{\mathbf{e}}_{2}\right)=\left(\hat{\mathbf{e}}_{\theta}, \hat{\mathbf{e}}_{\phi}\right)$. We consider the electron and baryon bulk velocity directions as $\mathbf{v}_{e}=\mathbf{v}_{b} \| \mathbf{K}$ and the photon polarization vectors are taken to be

$\hat{\varepsilon}_{1 x}=\cos \theta \cos \varphi, \hat{\varepsilon}_{1 y}=\cos \theta \sin \varphi, \hat{\varepsilon}_{1 z}=-\sin \theta$,

$\hat{\varepsilon}_{2 x}=-\sin \varphi, \hat{\varepsilon}_{2 y}=\cos \varphi, \hat{\varepsilon}_{2 z}=0$.

Meanwhile, temperature anisotropy (I) and polarization $(\mathrm{Q}, \mathrm{U})$ of the $\mathrm{CMB}$ radiation can be expanded in an appropriate spin-weighted basis as follows [31,32]:

$$
\begin{aligned}
\Delta_{I}^{(S)}(\mathbf{K}, \mathbf{k}, \tau) & =\sum_{\ell m} a_{\ell m}(\tau, K) Y_{\operatorname{lm}}(\hat{\mathbf{n}}), \\
\Delta_{P}^{ \pm(S)}(\mathbf{K}, \mathbf{k}, \tau) & =\sum_{\ell m} a_{ \pm 2, \ell m}(\tau, K)_{ \pm 2} Y_{\operatorname{lm}}(\hat{\mathbf{n}}),
\end{aligned}
$$

and

$$
\begin{aligned}
\Delta_{I}^{(S)}(\mathbf{K}, \mathbf{k}, \tau) & =\left(4 k \frac{\partial I_{0}}{\partial k}\right)^{-1} \Delta_{I}^{(S)}(\mathbf{K}, \mathbf{k}, \tau), \\
\Delta_{P}^{ \pm(S)} & =Q^{(S)} \pm i U^{(S)}
\end{aligned}
$$

For each plane wave, the scattering can be described as the transport through a plane parallel medium [33,34], which leads to the Boltzmann equations as

$$
\begin{aligned}
& \frac{\mathrm{d}}{\mathrm{d} \tau} \Delta_{I}^{(S)}+i K \mu \Delta_{I}^{(S)}+4[\dot{\psi}-i K \mu \varphi]=C_{e \gamma}^{I}, \\
& \frac{\mathrm{d}}{\mathrm{d} \tau} \Delta_{P}^{ \pm(S)}+i K \mu \Delta_{P}^{ \pm(S)}=C_{e \gamma}^{ \pm}-i v_{b} \kappa_{\mathrm{NC}}^{ \pm} \Delta_{V}^{(S)},
\end{aligned}
$$

and

$$
\begin{aligned}
& \frac{\mathrm{d}}{\mathrm{d} \tau} \Delta_{V}^{(S)}+i K \mu \Delta_{V}^{(S)}=C_{e \gamma}^{V}+i \frac{v_{b}}{2} \\
& \quad \times\left[\kappa_{\mathrm{NC}}^{-} \Delta_{P}^{+(S)}+\kappa_{\mathrm{NC}}^{+} \Delta_{P}^{-(S)}\right],
\end{aligned}
$$

here $C_{e \gamma}^{I}, C_{e \gamma}^{ \pm}$, and $C_{e \gamma}^{V}$ indicate the contributions from the usual photon-electron Compton scattering to the time evolution of the $I, \Delta_{P}^{ \pm(S)}$, and $V$ parameters, respectively; their expressions can be found for example in [2,31,32]. In (51) (53) $\mu=\hat{\mathbf{n}} \cdot \hat{\mathbf{K}}=\cos \theta, \theta$ is the angle between the direction 
of the CMB photon $\hat{\mathbf{n}}=\mathbf{k} /|\mathbf{k}|$ and the wave-vectors $\mathbf{K}$, and

$\kappa_{\mathrm{NC}}=a(\tau) \frac{3}{4} \frac{\sigma^{T}}{\alpha} \frac{m_{e}^{2}}{\Lambda^{2}} \bar{n}_{e} \sum_{f=e, p} \frac{m_{f}}{k^{0}}, \quad \kappa_{\mathrm{NC}}^{ \pm}=\kappa_{\mathrm{NC}}(C \pm i D)$,

where $C$ and $D$ are given in (41) and $a(\tau)$ is the normalized scaling factor. The values of $\Delta_{P}^{ \pm(S)}(\hat{\mathbf{n}})$ and $\Delta_{V}^{(S)}$ at the present time $\tau_{0}$ and the direction $\hat{\mathbf{n}}$ are obtained by integrating the Boltzmann equation (53) along the line of sight [31,32] and summing over all the Fourier modes $K$ as follows:

$$
\begin{aligned}
\Delta_{P}^{ \pm(S)}(\hat{\mathbf{n}}) & =\int \mathrm{d}^{3} \mathbf{K} \xi(\mathbf{K}) e^{ \pm 2 i \phi_{K, n}} \Delta_{P}^{ \pm(S)}\left(\mathbf{K}, \mu, \tau_{0}\right), \\
\Delta_{V}^{(S)}(\hat{\mathbf{n}}) & =\int \mathrm{d}^{3} \mathbf{K} \xi(\mathbf{K}) \Delta_{V}^{(S)}\left(\mathbf{K}, \mu, \tau_{0}\right),
\end{aligned}
$$

where $\phi_{K, n}$ is the angle needed to rotate the $\mathbf{K}$ - and $\hat{\mathbf{n}}$ dependent basis to a fixed frame in the sky, $\xi(\mathbf{K})$ is a random variable used to characterize the initial amplitude of the mode, and

$$
\begin{aligned}
\Delta_{P}^{ \pm(S)}\left(\mathbf{K}, \mu, \tau_{0}\right)= & \int_{0}^{\tau_{0}} \mathrm{~d} \tau \dot{\tau}_{e \gamma} e^{i x \mu-\tau_{e \gamma}} \\
& \times\left[\frac{3}{4}\left(1-\mu^{2}\right) \Pi(K, \tau)-i v_{b} \frac{\kappa_{\mathrm{NC}}^{ \pm}}{\dot{\tau}_{e \gamma}} \Delta_{V}^{(S)}\right]
\end{aligned}
$$

and

$$
\begin{aligned}
\Delta_{V}^{(S)}\left(\mathbf{K}, \mu, \tau_{0}\right)= & \frac{1}{2} \int_{0}^{\tau_{0}} \mathrm{~d} \tau \dot{\tau}_{e \gamma} e^{i x \mu-\tau_{e \gamma}}\left[3 \mu \Delta_{V 1}^{(S)}\right. \\
& \left.+i v_{b}\left(\frac{\kappa_{\mathrm{NC}}^{-}}{\dot{\tau}_{e \gamma}} \Delta_{P}^{+(S)}+\frac{\kappa_{\mathrm{NC}}^{+}}{\dot{\tau}_{e \gamma}} \Delta_{P}^{-(S)}\right)\right], \\
\approx & \frac{1}{2} \int_{0}^{\tau_{0}} \mathrm{~d} \tau \dot{\tau}_{e \gamma} e^{i x \mu-\tau_{e \gamma}}\left[3 \mu \Delta_{V 1}^{(S)}\right. \\
& \left.+2 i v_{b} C \frac{\kappa_{\mathrm{NC}}}{\dot{\tau}_{e \gamma}} \Delta_{P}^{(S)}\right],
\end{aligned}
$$

in which $x=K\left(\tau_{0}-\tau\right), C$ is defined in (41) and

$$
\Delta_{P}^{(S)}(\mathbf{K}, \mu, \tau)=\int_{0}^{\tau} \mathrm{d} \tau \dot{\tau}_{e \gamma} e^{i x \mu-\tau_{e \gamma}}\left[\frac{3}{4}\left(1-\mu^{2}\right) \Pi(K, \tau)\right]
$$

where

$$
\Pi=\Delta_{T 2}^{(S)}+\Delta_{P 2}^{(S)}+\Delta_{P 0}^{(S)} .
$$

The differential optical depth $\dot{\tau}_{e \gamma}(\tau)$ and total optical depth $\tau_{e \gamma}(\tau)$ due to the Thomson scattering at time $\tau$ are defined as

$\dot{\tau}_{e \gamma}=a n_{e} \sigma_{T}, \quad \tau_{e \gamma}(\tau)=\int_{\tau}^{\tau_{0}} \dot{\tau}_{e \gamma}(\tau) \mathrm{d} \tau$.
As is shown in (51), the temperature anisotropy $\Delta_{I}^{(S)}$ does not have any source due to forward Compton scattering in $\mathrm{NC}$ space-time; therefore, we only focus on the other equations to explore the NC effects. Meanwhile, (55) and (56) indicate that the effect of non-commutativity on the linear and circular polarization can be valuable for a significant value of $\frac{\kappa_{\mathrm{NC}}}{\dot{\bar{t}}_{e \gamma}}$, which is defined as follows:

$\tilde{\kappa}=\frac{\kappa_{\mathrm{NC}}}{\dot{\tau}_{e \gamma}}=\frac{3}{4} \frac{1}{\alpha} \frac{m_{e}^{2}}{\Lambda^{2}} \sum_{f=e, p} \frac{m_{f}}{k^{0}}$,

which leads to larger values for protons than electrons.

\section{CMB power spectrum in NC space-time}

In the preceding section we found that Compton scattering in the NC space changes the Boltzmann equations for the time evolution of the polarized CMB photons. Here, we are ready to find the power spectra of $I, B, E$, and $V$ in the $\mathrm{NC}$ background. To this end, we consider the power spectrum,

$C_{X l}=\frac{1}{2 l+1} \sum_{m}\left\langle a_{X, \operatorname{lm}}^{*} a_{X, \operatorname{lm}}\right\rangle, \quad X=\{I, E, B, V\}$,

where

$a_{E, \mathrm{~lm}}=-\left(a_{2, \mathrm{~lm}}+a_{-2, \mathrm{~lm}}\right) / 2$,
$a_{B, \operatorname{lm}}=i\left(a_{2, \mathrm{~lm}}-a_{-2, \mathrm{~lm}}\right) / 2$,
$a_{V, \mathrm{~lm}}=\int \mathrm{d} \Omega Y_{\operatorname{lm}}^{*} \Delta_{V}$,

which for the circularly polarized part of the CMB photons by using (56) in the power spectrum $C_{\mathrm{Vl}}$ leads to

$$
\begin{aligned}
C_{\mathrm{Vl}}= & \frac{1}{2 l+1} \sum_{m}\left\langle a_{V, \operatorname{lm}}^{*} a_{V, \operatorname{lm}}\right\rangle, \\
\approx & \frac{1}{2 l+1} \int \mathrm{d}^{3} \mathbf{K} P_{v}(\mathbf{K}) \sum_{m} \\
& \times\left|\int \mathrm{d} \Omega Y_{\operatorname{lm}}^{*} \int_{0}^{\tau_{0}} \mathrm{~d} \tau \dot{\tau}_{e \gamma} e^{i x \mu-\tau_{e \gamma}} C \tilde{\kappa} \Delta_{P}^{(S)}\right|^{2},
\end{aligned}
$$

where

$P_{v}(\mathbf{K}) \delta\left(\mathbf{K}^{\prime}-\mathbf{K}\right)=\left\langle\left(\xi(\mathbf{K}) v_{b}\right)\left(\xi\left(\mathbf{K}^{\prime}\right) v_{b}\right)\right\rangle$,

and $P_{v}(\mathbf{K})$ is the velocity power spectrum, which can be expressed in terms of the primordial scalar spectrum $P_{\phi}^{(S)}$ as [35]

$P_{v}(\mathbf{K}, \tau) \sim P_{\phi}^{(S)}(\mathbf{K}, \tau)$

Indeed the effect of non-commutativity on the scalar power spectrum should be considered; it is investigated in [36,37], but the point is that we are going to calculate the leading 
order of non-commutative effects on the circular and Bmode polarizations, which is the order of $\theta^{2}$. Furthermore we cannot generate the B-mode and circular polarization in the scalar perturbation without considering the effects of non-commutativity on the interactions (see previous section). If we consider the non-commutative corrections due to the interaction and power spectrum altogether for the circular and B-mode polarizations, our results depend at least on $\theta^{3}$, which is not the leading order term. Now (67) and (70) can provide an estimate on $C_{\mathrm{Vl}}$ in terms of the linearly polarized power spectrum $C_{\mathrm{Pl}}$ as follows:

$\tilde{\kappa}_{\min }^{2} C_{\mathrm{Pl}} \leq C_{\mathrm{Vl}} \leq \tilde{\kappa}_{\max }^{2} C_{\mathrm{Pl}}$

where

$\tilde{\kappa}_{\max }=\frac{3}{4} \frac{m_{e}+m_{p}}{T^{0}} \frac{1}{\alpha} \frac{m_{e}^{2}}{\Lambda^{2}} \simeq 3 \times 10^{-1}(20 \mathrm{TeV} / \Lambda)^{2}$,

$\tilde{\kappa}_{\min }=\frac{3}{4} \frac{m_{e}+m_{p}}{T^{\mathrm{lss}}} \frac{1}{\alpha} \frac{m_{e}^{2}}{\Lambda^{2}} \simeq 3 \times 10^{-4}(20 \mathrm{TeV} / \Lambda)^{2}$,

in which $k^{0}=T^{0}$ and $k^{0}=T^{\text {lss }}$ are the energies of the CMB photons at the present time and the last scattering epoch, respectively. Using the experimental value for the linearly polarized power spectrum of the CMB photons, which is of the order of $0.1 \mu K^{2}$ for $l<250$ [38,39], one finds from (71)-(73) an estimation on the range of $C_{\mathrm{Vl}}$,

$0.01 n K^{2} \leq C_{\mathrm{Vl}} \leq 0.01 \mu K^{2}$

for a conservative value of the NC scale of the order of $\Lambda \sim$ $20 \mathrm{TeV}$, the lower bound is reported in [22] based on the CMB experiment. Meanwhile, for the more or less accepted value $\Lambda \sim 1 \mathrm{TeV}$ [40-43], the circular polarization power spectrum $C_{\mathrm{Vl}}$ can be obtained in the following range:

$10^{-3} \mu K^{2} \leq C_{\mathrm{V} 1} \leq 10^{3} \mu K^{2}$,

which is in the range of achievable experimental values.

In addition to $C_{\mathrm{Vl}}$, Compton scattering in the $\mathrm{NC}$ space, in contrast with ordinary space, can also generate the B-mode polarization. To explore such a property we give the CMB polarization in terms of the divergence-free part (B-mode $\left.\Delta_{B}^{(S)}\right)$ and the curl-free part (E-mode $\left.\Delta_{E}^{(S)}\right)$, which are defined as

$\Delta_{E}^{(S)}(\hat{\mathbf{n}}) \equiv-\frac{1}{2}\left[\bar{\partial}^{2} \Delta_{P}^{+(S)}(\hat{\mathbf{n}})+\check{\partial}^{2} \Delta_{P}^{-(S)}(\hat{\mathbf{n}})\right]$,

$\Delta_{B}^{(S)}(\hat{\mathbf{n}}) \equiv \frac{i}{2}\left[\overline{\widetilde{\partial}}^{2} \Delta_{P}^{+(S)}(\hat{\mathbf{n}})-\check{\partial}^{2} \Delta_{P}^{-(S)}(\hat{\mathbf{n}})\right]$,

where $\partial$ and $\bar{\partial}$ are spin raising and lowering operators, respectively [31,32]. As Eqs. (56), (77), and (63) show the B-mode power spectrum $C_{\mathrm{Bl}}$ due to the forward electron and proton
Compton scattering in the NC space-time depends on the circular polarization power spectrum which can be estimated to be

$C_{\mathrm{Bl}}^{S} \propto \overline{\tilde{\kappa}}^{2} C_{\mathrm{Vl}}, \quad \tilde{\kappa}_{\min }<\overline{\tilde{\kappa}}<\tilde{\kappa}_{\max }$,

where $S$ indicates the scalar mode of the matter perturbation. Furthermore, the B-mode power spectrum depends on the scale of the NC parameter $\Lambda$, through $\overline{\tilde{\kappa}}$, which can have a significant effect on the value of the $r$ parameter even for $\Lambda \sim 20 \mathrm{TeV}$. In fact, by using Eqs. (71) and (78) one has

$\tilde{\kappa}_{\min }^{4} C_{\mathrm{Pl}} \leq C_{\mathrm{Bl}}^{S} \leq \tilde{\kappa}_{\max }^{4} C_{\mathrm{Pl}}$,

which for $\Lambda \sim 20 \mathrm{TeV}$ leads to

$10^{-4} p K^{2} \leq C_{\mathrm{B} 1}^{S} \leq 10^{-4} \mu K^{2}$

and

$10 n K^{2} \leq C_{\mathrm{B} 1}^{S} \leq 10 m K^{2}$

for $\Lambda \sim 1 \mathrm{TeV}$, which is comparable with the value of $C_{\mathrm{Bl}}^{\mathrm{ob}} \sim 0.01 \mu K^{2}$ for $l<250[38,39]$.

\section{Conclusion}

We considered the time evolution of the Stokes parameters in the NC space-time. We showed that the NC corrections on Compton scattering can lead to the circular polarization for the $\mathrm{CMB}$ radiation. It is also shown that the $\mathrm{B}$-mode spectrum in contrast with the usual production via the tensor mode perturbation, can be generated by the scalar mode perturbation in the NC space-time. The obtained result shows that to fully understand the origin of the reported $r$ parameter $[12,38,39]$, one should consider all the alternative sources for the B-mode spectrum of polarized CMB photons. Furthermore, we found the $\mathrm{V}$-mode power spectrum in the range of nano-kelvin squared and higher for the NC scale of 1$20 \mathrm{TeV}$, which is in the range of the accuracy of observational data $[38,39,44-46]$. We also showed that the B-mode power spectrum $C_{\mathrm{Bl}}$, due to forward electron and proton Compton scattering in the NC space-time, depends on the circular polarization power spectrum for the scalar mode of the matter perturbation.

Open Access This article is distributed under the terms of the Creative Commons Attribution 4.0 International License (http://creativecomm ons.org/licenses/by/4.0/), which permits unrestricted use, distribution, and reproduction in any medium, provided you give appropriate credit to the original author(s) and the source, provide a link to the Creative Commons license, and indicate if changes were made.

Funded by $\mathrm{SCOAP}^{3}$. 


\section{References}

1. J.D. Jackson, Classical Electrodynamic (Wiley, New York, 1998)

2. A. Kosowsky, Ann. Phys. 246, 49 (1996). arXiv:astro-ph/9501045

3. W. Hu, M.J. White, N. Astron. 2, 323 (1997). arXiv:astro-ph/9706147

4. A. Cooray, A. Melchiorri, J. Silk, Phys. Lett. B 554, 1 (2003). arXiv:astro-ph/0205214

5. M. Giovannini (2002). arXiv:hep-ph/0208152

6. M. Giovannini, K.E. Kunze, Phys. Rev. D 78, 023010 (2008). arXiv:0804.3380 [astro-ph]

7. E. Bavarsad, M. Haghighat, Z. Rezaei, R. Mohammadi, I. Motie, M. Zarei, Phys. Rev. D 81, 084035 (2010). arXiv:0912.2993 [hep-th]

8. R. Mohammadi, Eur. Phys. J. C 74(10), 3102 (2014). arXiv: 1312.2199 [astro-ph.CO]

9. R. Mohammadi, S.-S. Xue, Phys. Lett. B 731, 272 (2014). arXiv:1312.3862 [hep-ph]

10. I. Motie, S.-S. Xue, Europhys. Lett. 100, 17006 (2012). arXiv: 1104.3555 [hep-ph]

11. R.F. Sawyer. arXiv:1205.4969 [astro-ph.CO]

12. P.A.R. Ade et al. [BICEP2 Collaboration]. arXiv:1403.3985 [astroph.CO]

13. P.A.R. Ade et al. [BICEP2 and Keck Array Collaborations]. arXiv:1510.09217 [astro-ph.CO]

14. C. Scoccola, D. Harari, S. Mollerach, Phys. Rev. D 70, 063003 (2004)

15. L. Cam-panelli, A.D. Dolgov, M. Giannotti, F.L. Villante, Astrophys. J. 616, 1 (2004)

16. A. Kosowsky, T. Kahniashvili, G. Lavrelashvili, B. Ratra, Phys. Rev. D 71, 043006 (2005)

17. L. Pogosian, A.P.S. Yadav, Y.-F. Ng, T. Vachaspati, Phys. Rev. D 84, 043530 (2011)

18. C. Bonvin, R. Durrer, R. Maartens, Phys. Rev. Lett. 112, 191303 (2014). arXiv:1403.6768 [astro-ph.CO]

19. M. Giovannini, Phys. Rev. D 90, 041301 (2014). arXiv:1404.3974 [astro-ph.CO]

20. J. Khodagholizadeh, R. Mohammadi, S.-S. Xue, Phys. Rev. D 90, 091301 (2014). arXiv:1406.6213 [astro-ph.CO]

21. R. Mohammadi, J. Khodagholizadeh, M. Sadegh, S.S. Xue. arXiv: 1602.00237 [astro-ph.CO]

22. P.K. Joby, P. Chingangbam, S. Das, Phys. Rev. D 91(8), 083503 (2015). arXiv:1412.6036 [astro-ph.CO]

23. F. Ardalan, H. Arfaei, M.M. Sheikh-Jabbari, JHEP 9902, 016 (1999). arXiv:9810072
24. N. Seiberg, E. Witten, JHEP 9909, 032 (1999). arXiv:9908142

25. X. Calmet, B. Jurco, P. Schupp, J. Wess, M. Wohlgenannt, Eur. Phys. J. C 23, 363 (2002)

26. M. Chaichian, P. Presnajder, M.M. Sheikh-Jabbari, A. Tureanu. arXiv:0107037

27. M. Chaichian, P. Presnajder, M.M. Sheikh-Jabbari, A. Tureanu. arXiv:0107055

28. I.F. Riad, M.M. Sheikh-Jabbari, JHEP 0008, 045 (2000). arXiv:hep-th/0008132

29. M. Chaichian, M.M. Sheikh-Jabbari, A. Tureanu, Phys. Rev. Lett. 86, 2716 (2001)

30. B. Melic, K. Passek-Kumericki, J. Trampetic, P. Schupp, M. Wohlgenannt, Eur. Phys. J. C 42, 483 (2005). arXiv:0502249

31. M. Zaldarriaga, U. Seljak, Phys. Rev. D 55, 1830 (1997). arXiv:astro-ph/9609170

32. M. Zaldarriaga, D.N. Spergel, U. Seljak, Astrophys. J. 488, 1 (1997). arXiv:astro-ph/9702157

33. S. Chandrasekhar, Radiative Transfer (Dover, New York, 1960)

34. N. Kaiser, Mon. Not. R. Astron. Soc. 202, 1169 (1983)

35. D.S. Gorbunov, V.A. Rubakov, Introduction to the Theory of the Early Universe Cosmological Perturbations and Inflationary Theory. World Scientific Publishing Company Incorporated, Singapore (2011)

36. E. Akofor, A.P. Balachandran, S.G. Jo, A. Joseph, B.A. Qureshi, J. High Energy Phys. 05, 092 (2008)

37. E. Akofor, A.P. Balachandran, A. Joseph, L. Pekowsky, B.A. Qureshi, Phys. Rev. D 79, 063004 (2009)

38. Planck Collaboration I (2013). arXiv:planck2013-p01,p08

39. P.A.R. Ade et al. [Planck Collaboration]. arXiv:1502.02114 [astroph.CO]

40. M. Haghighat, M. Khorsandi, Eur. Phys. J. C 75, 4 (2015). arXiv: 1410.0836 [hep-ph]

41. M. Haghighat, N. Okada, A. Stern, Phys. Rev. D 82, 016007 (2010). arXiv:1006.1009 [hep-ph]

42. A. Prakash, A. Mitra, P.K. Das, Phys. Rev. D 82, 055020 (2010)

43. M.M. Ettefaghi, Phys. Rev. D 86, 085038 (2012)

44. M.D. Niemack, P.A.R. Ade, J. Aguirre etal., Proc. SPIE 7741 (2010). arXiv:1006.5049 [astro-ph.IM]

45. A. Kogut et al. (2011). arXiv:1105.2044 [astro-ph.CO]

46. J.P. Filippini, P.A.R. Ade et al. (2011). arXiv:1106.2158 [astroph.CO] 